\title{
Variation among Beef Bulls in the Ratio of X- to Y-Chromosome Bearing Spermatozoa
}

\author{
Rick W. Rorie ${ }^{1}$, Pedro A. Delgado',2, Toby D. Lester ${ }^{1}$ \\ ${ }^{1}$ Department of Animal Science, University of Arkansas, Fayetteville, USA \\ 2"MVZ-ZIP-UPEA”, “INZ-T-UCB”, El Alto, Bolivia \\ Email: rrorie@uark.edu
}

Received 29 July 2014; revised 30 August 2014; accepted 13 September 2014

Copyright (C) 2014 by authors and Scientific Research Publishing Inc.

This work is licensed under the Creative Commons Attribution International License (CC BY).

http://creativecommons.org/licenses/by/4.0/

(c) (i) Open Access

\begin{abstract}
A study was conducted to evaluate variation in the ratio of $X$ - to $Y$-bearing sperm of individual ejaculates and to determine any relationship between skewed sex ratio and either routine morphological evaluation or computer assisted sperm analysis (CASA). Semen was collected from bulls weekly for 6 consecutive weeks, sperm DNA was recovered and quantitative, and real-time PCR was used to determine the ratio of $X$ - to Y-bearing sperm in each ejaculate. The overall mean of $X$-bearing sperm within ejaculates was $54.7 \%$ over the 6 weeks of semen collections. The percentages of $X$-bearing sperm were similar $(P>0.5)$ across all collections. Between bulls, there were differences $(P<0.05)$ in the mean percentage of $X$-bearing sperm. No significant correlations were found between CASA parameters and percentage of $\mathrm{X}$-bearing sperm across bulls, so analysis was done within each bull. Different combinations of CASA and/or morphological parameters were found to correlate with the percentage of $X$-bearing sperm but the prediction equations were specific for individual bulls and unlikely to be of use across bulls. These results confirm that the ratio of X- to Y-bearing sperm may be skewed in some ejaculates of bull semen. Some sperm parameters measured by CASA or routine morphological evaluation were associated with semen sex ratio.
\end{abstract}

\section{Keywords}

Sex Ratio, Spermatozoa, Ejaculates, Semen, Bulls

\section{Introduction}

Sex or gender ratio can be defined as the proportion of males to females in a given population. Numerous factors have been attributed to altered sex ratio, including nutrition, season, disease, gonadotropin and steroid hormone

*Corresponding author.

How to cite this paper: Rorie, R.W., Delgado, P.A. and Lester, T.D. (2014) Variation among Beef Bulls in the Ratio of X- to Y-Chromosome Bearing Spermatozoa. Advances in Reproductive Sciences, 2, 69-75.

http://dx.doi.org/10.4236/arsci.2014.24008 
levels, time of insemination, social status, stress, age and parity [1]-[3]. Physiologically, an altered sex ratio might be achieved maternally by facilitating or inhibiting the transport of either X- or Y-bearing sperm through the reproductive tract, preferential selection of sperm at fertilization, or sex-specific death of embryos after fertilization [2]. Another possible means of altering the sex ratio is altered ratio of X- or Y-bearing sperm at ejaculation.

At the completion of spermatogenesis, the ratio of X- to Y-chromosome bearing sperm would be expected to be 1:1. However, Chandler et al. [4] reported that Y-bearing sperm within ejaculates of bull semen varied from 24\% to $84 \%$. In a later study, Chandler et al. [5] measured the proportion of Y-bearing sperm in 36 lots (ejaculates) of Holstein semen and then correlated the predicted with actual sex ratio of calves born after insemination with the various semen lots. Results of the study indicated a significant variation in the proportion of Y-bearing sperm among lots of semen, which positively correlated $(r=0.82)$ to male calves born. In another study, Checa et al. [6] reported the X-chromosome content varied from $~ 39 \%$ to $58 \%$ in ejaculates of bull semen. However the variation was attributed to individual bulls and not to ejaculates within bulls. The present study was conducted to confirm that the ratio of $\mathrm{X}$ - to Y-chromosome bearing sperm could be skewed in individual ejaculates of bull semen. Semen collections were also analyzed, using routine morphological evaluation and computer assisted sperm analysis (CASA), to determine if any skewed sex ratio might be reflected in measurable sperm parameters. Previous studies [7] [8] have shown that sperm motility parameters measured by CASA are accurate and repeatable, while eliminating technician-to-technician variation. If CASA motility parameters alone or in combination with morphology could be used to identify ejaculates of semen with skewed sex ratios, it would expand the ability to alter the gender of offspring in cattle.

\section{Material and Methods}

\subsection{Animals, Semen Collection and Evaluation}

Six Angus bulls ranging from 3 to 10 years of age were used for the study. Semen was collected by electroejaculation weekly for 6 consecutive weeks. Semen samples were placed into a $35^{\circ} \mathrm{C}$ water bath immediately after collection for transport to the laboratory. A computer assisted system analysis (CASA) system (Hamilton-Thorne IVOS, Beverly MA) with version 12 TOX IVOS software was used for sperm analysis within $1 \mathrm{~h}$ of collection. Sperm motility analysis parameters were measured, using the default analysis settings recommended by Hamilton-Thorne for bull semen. For analysis, semen samples were diluted to $\sim 25 \times 10^{6} \mathrm{sperm} / \mathrm{ml}$ with Tyrode's albumin-lactate-pyruvate (TALP) medium and placed in a 2X-CEL slide (Hamilton-Thorne) with a fixed $20 \mu \mathrm{m}$ depth. Ten fields were scanned down the entire length of the slide, with 30 video frames captured per field at a frame rate of $60 \mathrm{~Hz}$. A minimum of 400 sperm was counted in each sample. Each week, variables measured with CASA were motility (\%), progressive motility (\%), velocity distribution (rapid, medium and slow \%), path velocity (VAP $\mu \mathrm{m} / \mathrm{s}$ ), progressive velocity (VSL $\mu \mathrm{m} / \mathrm{s}$ ), track speed (VCL $\mu \mathrm{m} / \mathrm{s}$ ), lateral amplitude (ALH $\mu \mathrm{m})$, beat cross frequency (BCF Hz), straightness (STR \%), linearity (LIN \%), and sperm head size (Area, $\mu \mathrm{m}$ ).

Immediately after CASA analysis, a slide was prepared of each semen sample for morphological analysis, using eosin-nigrosin live/dead stain [9]. Briefly, equal portions (25 $\mu \mathrm{l} \mathrm{each)} \mathrm{of} \mathrm{stain} \mathrm{and} \mathrm{semen} \mathrm{were} \mathrm{mixed,} \mathrm{then}$ smeared the length of a slide and immediately dried on a slide warmer. Sperm morphology was subjectively evaluated by means of light microscope at $400 \times$. A total of 200 sperm were counted per slide. Morphology evaluated was the percent live, major defects (pyriform heads, proximal drops, strongly coiled, and cratered heads), and minor defects (bend or coiled tails, tailess, small or giant heads).

\subsection{Extraction and Quantification of DNA from Sperm}

The sperm DNA extraction protocol was performed using the procedure described by Heyen et al. [10] with minor modifications. Semen samples $(1 \mathrm{ml})$ were centrifuged at $5000 \mathrm{xg}$ for $5 \mathrm{~min}$, and seminal plasma was removed. The sperm pellet was washed by re-suspension in $1 \mathrm{ml}$ of D-PBS and followed by centrifugation at $5000 \mathrm{xg}$ for 5 min. After removal of supernatant, $450 \mu \mathrm{l}$ of pre-warmed $\left(60^{\circ} \mathrm{C}\right)$ extraction buffer consisting of $10 \mathrm{mM}$ Tris base (pH 8.0), $10 \mathrm{mM}$ EDTA, $1 \%$ SDS and $100 \mathrm{mM} \mathrm{NaCl}$ in ultrapure water was added to each sperm sample, followed by $50 \mu \mathrm{l}$ of $0.8 \mathrm{M}$ DL-dithiothreitol (final concentration of $80 \mathrm{mM}$ ) and $200 \mu \mathrm{g}$ of Proteinase K (Invitrogen, Eugene, OR; specific activity $>20$ units/mg). Sperm samples were then incubated overnight $(\sim 15 \mathrm{~h})$ at $60^{\circ} \mathrm{C}$ in a block heater. 
After incubation, samples were centrifuged at 15,000 xg for $10 \mathrm{~min}$. The supernatant was removed and placed into a $1.7 \mathrm{ml}$ centrifuge tube. Next, $100 \mu \mathrm{l}$ of saturated $\mathrm{NaCl}(5 \mathrm{M})$ was added to each tube and shaken for $2 \mathrm{~min}$, then $1 \mathrm{ml}$ of absolute ethanol was added to precipitate the DNA. The tubes were centrifuged for 10 minutes at $15,000 \mathrm{xg}$ to pellet the DNA and supernatant was discarded. The DNA pellet was washed with $70 \%$ ethanol and centrifuged again before pouring off the ethanol and allowing the tubes to dry at room temperature. The DNA samples were re-suspended in $200 \mu \mathrm{l}$ TE buffer $(10 \mathrm{mM}$ Tris-HCl and $1 \mathrm{mM}$ EDTA in PCR water). The DNA samples were stored at $-20^{\circ} \mathrm{C}$ until DNA quantification. Sperm DNA concentration was quantified by means of Quant-iT fluorometer (Invitrogen) using a Quant-iT dsDNA HS Assay Kit (Cat. No. Q32854). Working stock solutions of $5 \mathrm{ng} / \mu \mathrm{l}$ sperm DNA were prepared in PCR water and stored in the refrigerator $\left(4^{\circ} \mathrm{C}\right)$ until use.

\subsection{Quantitative Real Time PCR (qRT-PCR)}

The sex ratio of individual ejaculates of semen was determined using the relative standard curve method of qRT-PCR using a StepOnePlus Real-Time PCR System (Applied Biosystems, Beverly MA). The ratio was based on amplification of $\mathrm{Y}$ and X-chromosome specific fragments of Sry and coagulation Factor IX, respectively [5]. Forward (5'-CGT CAA GCG ACC CAT GAA C-3') and reverse (5'-GAG CCA CCT TTC GTC TTC GTT-3') primers were used to amplify a 62 bp fragment of the Bos taurus sex determining region Y protein (Sry) gene (Genbank: AF148462). A FAM-labeled internal (5'- CTT CAT TGT GTG GTC TCG T-3') TaqMan probe was used to detect the resulting pcr product.

Forward (5'-CTG CCC ATC AGG CAT TGT AGT-3') and reverse (5'-GAT GGC AGG TGA GGG TAG GA-3) primers were also used to amplify a 63 bp fragment of Bos taurus coagulation factor IX gene (Genbank: BC149563) on the X chromosome. A VIC-labeled internal (5'-CAG CGG AGC AAC TC -3') TaqMan probe was used to detect the resulting pcr product. All primers were ordered from Invitrogen. TaqMan probes were ordered from Applied Biosystems. Primer Express 3.0 software (Applied Biosystems) was used to design all primers and probes. Basic Local Alignment Search Tool (BLAST) available from the National Center for Biotechnology Information (www.ncbi.nlm.nih.gov) was used to confirm uniqueness of primer sequences. Both sets of primers were confirmed to amplify a single PCR product of the appropriate size prior to use in qRT-PCR.

The PCR reaction mix consisted of $12.5 \mu \mathrm{l}$ of 2x Universal Master Mix (Applied Biosystems), $900 \mathrm{nM}$ each of the appropriate forward and reverse primers, $200 \mathrm{nM}$ of the appropriate TaqMan probe and $25 \mathrm{ng}$ of template DNA in a volume of $25 \mu \mathrm{l}$. The amplification protocol (specified by Applied Biosystems) consisted of 2 min at $50^{\circ} \mathrm{C}, 10 \mathrm{~min}$ at $95^{\circ} \mathrm{C}$, followed by 40 cycles of $95^{\circ} \mathrm{C}$ for $15 \mathrm{sec}$ and $60^{\circ} \mathrm{C}$ for $1 \mathrm{~min}$. Within each 96 well reaction plate, $\mathrm{X}$ and $\mathrm{Y}$ standard curves (1:4 dilutions; 47.5, 23.75, 11.88, 5.94 and 2.97 ng of pooled male DNA) were run in triplicate. All samples were also run in triplicate, in addition the appropriate $\mathrm{X}$ and Y-specific primers and probes without sperm DNA template (negative control). For quantification, the Y-specific standard curve was used, with a triplicate sample of $25 \mathrm{ng}$ of DNA pooled from 6 bulls serving as the endogenous control. The reference sample was the pooled male DNA in triplicate.

\subsection{Statistical Analysis}

The mixed procedure of SAS (SAS Institute) was used to determine the effects of bull and collection within bull on the ratio of X-chromosome bearing sperm in ejaculates of semen. Component analysis was used to identify highly correlated CASA sperm parameters that could be combined into a single parameter. Within bulls, step-wise regression was used to determine correlations between sperm parameters measured by CASA or sperm morphology, and the ratio of $\mathrm{X}$-chromosome bearing sperm in semen ejaculates.

\section{Results}

\subsection{Quantitative Real Time PCR}

Initially, attempts were made to run a duplex qRT-PCR where both the Sry and Factor IX products were amplified within the same reaction. However, this altered amplification efficiency resulting in over-amplification of the Sry product and under-amplification of the Factor IX product. Therefore, simplex qRT-PCR was used. Because separate Sry and Factor IX standard curves, endogenous and negative controls, and separate amplification of Sry and Factor IX product for each sample, only 9 samples could be run per 96-well assay plate. Therefore, bulls were selected at random and all 6 samples from one bull and 3 samples from another bull were assayed 
within a plate, requiring 4 assay plates for all samples. Between the assay plates the $\mathrm{R}^{2}$ for the Factor IX standard curves ranged from 0.997 to 1.0 with efficiencies ranging from $100 \%$ to $102.5 \%$. For the Sry standard curve, the $\mathrm{R}^{2}$ ranged from 0.998 to 1.0 with efficiencies from $99.5 \%$ to $100.3 \%$.

\subsection{Variation in the Ratio of X- to Y-Chromosome Bearing Sperm in Ejaculates}

The overall mean of $X$-bearing sperm within ejaculates was $54.7 \% \pm 1.4 \%$, with a range from $43.5 \%$ to $61.5 \%$ over the 6 weeks of semen collections (Table 1$)$. The percentages of $X$-bearing sperm were similar $(P=0.528)$ across all collections. Across bulls, there were differences $(\mathrm{P}<0.05)$ in the mean percentage of $\mathrm{X}$-bearing sperm. The mean ratio of X-bearing sperm for bull $4(58.6 \%)$ was greater $(\mathrm{P}<0.05)$ that that for bull $2(52.1 \%)$ or 1 (52.7\%). The mean ratio of X-bearing sperm for the other 3 bulls ( 3,5 and 6 ) ranged from $53.3 \%$ to $56.6 \%$ was similar to the other bulls.

\subsection{Relationship of CASA or Morphology Parameters with \% X-Chromosome Bearing Sperm}

The means for sperm motility parameters measured over 6 weeks of semen collections are presented in Table 2 . Component analysis indicated that motile, progressive and rapid sperm were highly correlated $(r=0.93)$ so these parameters were averaged into a motility measure. Medium and slow sperm were found to be correlated $(r=0.80)$ so they were combined. Path (VAP and progressive (VSL) velocity $(r=0.95)$ were averaged as a velocity (VAP + VSL) measure. Point-to-point sperm track speed (VCL) and lateral sperm head oscillation (ALH) were averaged ( $\mathrm{r}$ $=0.93$ ) into a second velocity (VCL + ALH) measure. Straightness (STR) and linearity (LIN) which both measure the departure of sperm from a straight line were averaged $(r=0.82)$ into a sperm path $(\mathrm{STR}+\mathrm{LIN})$ measure. Beat frequency (BCF) and sperm head area (Area) were averaged $(r=0.80)$ as a measure of sperm head (BCF + Area) movement. Percent live sperm was correlated to motile $(r=0.81)$, progressive $(r=0.78)$ and rapid $(r=0.83)$ sperm but did not improve the measure. The percentage of major and minor abnormalities was only moderately correlated $(r=0.60)$, so the parameters were considered separately.

No significant correlations were found between CASA parameters and percentage of X-chromosome bearing sperm across bulls, so analysis was done within each bull. For bull 1 , the coefficient of determination $\left(\mathrm{R}^{2}\right)$ was $0.988(\mathrm{P}<0.01)$, for the regression equation: \% X-bearing sperm $=-22.822+(\mathrm{VAP}+\mathrm{VSL}) \times-0.167+(\mathrm{ALH}+$ $\mathrm{VCL}) \times 0.347+(\mathrm{STR}+\mathrm{LIN}) \times 0.830$. For bull 2 , the only parameter related to the $\% \mathrm{X}$-bearing sperm was $\%$ live sperm with the regression equation of $33.025+(\%$ live $) \times 0.253\left(\mathrm{R}^{2}=0.71 ; \mathrm{P}<0.05\right)$. Variation in the \% of X-bearing sperm in bull 5 was explained by the equation: $72.417+($ Med + Slow $) \times 0.591+($ VAP + VSL $) \times$ $0.2444+(\mathrm{STR}+\mathrm{LIN}) \times-0.01\left(\mathrm{R}^{2}=0.958 ; \mathrm{P}<0.05\right)$. For the remaining bulls $(3,4$ and 6$)$, none of the CASA or morphology parameters were correlated with the $\% \mathrm{X}$-bearing sperm within ejaculates of semen.

\section{Discussion}

Chandler et al. [4] compared the sex ratio of calves born following insemination with different lots (ejaculates) of semen collected from the same bulls. The results indicated that the percentage of X-bearing sperm in ejaculates varied from $\sim 24 \%$ to $84 \%$, and this variation contributed to the variation noted in the gender of calves born. In a follow-up study, Chandler et al. [5] confirmed these data, but reported that the proportion of X-bearing sperm

Table 1. Variation in the percentage of X-bearing sperm in beef bull ejaculates.

\begin{tabular}{|c|c|c|c|c|c|c|c|}
\hline \multicolumn{8}{|c|}{ Percentage of X-chromosome sperm by week of collection } \\
\hline Bull & 1 & 2 & 3 & 4 & 5 & 6 & LSMean \\
\hline 1 & 51.0 & 51.7 & 52.4 & 57.8 & 52.7 & 50.7 & $52.7^{\mathrm{b}}$ \\
\hline 2 & 55.0 & 52.3 & 50.0 & 52.4 & 56.8 & 45.9 & $52.1^{b}$ \\
\hline 3 & 53.3 & 56.8 & 52.9 & 43.5 & 58.6 & 54.7 & $53.3^{\mathrm{ab}}$ \\
\hline 4 & 58.0 & 58.7 & 57.9 & 59.1 & 61.5 & 56.2 & $58.6^{a}$ \\
\hline 5 & 54.8 & 56.3 & 54.2 & 52.6 & 56.4 & 58.3 & $55.4^{\mathrm{ab}}$ \\
\hline 6 & 53.0 & 56.9 & 53.6 & 58.8 & 58.5 & 58.7 & $56.6^{\mathrm{ab}}$ \\
\hline
\end{tabular}

Means with different superscripts within rows differ $(\mathrm{P}<0.05)$. 
Table 2. Mean \pm S.E. for sperm parameters measured by CASA over 6 weeks of semen collections.

\begin{tabular}{ccccccc}
\hline \multicolumn{7}{c}{ Bull number } \\
$\begin{array}{c}\text { Sperm } \\
\text { parameter }\end{array}$ & 1 & 2 & 3 & 4 & 5 & 6 \\
\hline Motile & $56.8 \pm 6.4$ & $63.0 \pm 9.4$ & $68.7 \pm 4.5$ & $42.7 \pm 5.3$ & $61.0 \pm 6.1$ & $70.0 \pm 10.3$ \\
Progressive & $46.2 \pm 5.4$ & $43.5 \pm 7.2$ & $48.7 \pm 4.9$ & $25.3 \pm 3.7$ & $40.5 \pm 7.3$ & $54.7 \pm 8.3$ \\
Rapid & $51.7 \pm 5.9$ & $57.5 \pm 9.2$ & $63.5 \pm 5.0$ & $36.0 \pm 5.3$ & $52.5 \pm 8.9$ & $67.5 \pm 10.6$ \\
Medium & $5.0 \pm 0.9$ & $5.5 \pm 0.8$ & $5.2 \pm 1.0$ & $6.7 \pm 0.9$ & $8.7 \pm 3.5$ & $2.5 \pm 0.7$ \\
Slow & $17.3 \pm 4.1$ & $8.5 \pm 1.0$ & $10.7 \pm 3.5$ & $15.5 \pm 2.4$ & $18.2 \pm 5.3$ & $5.7 \pm 1.5$ \\
VAP & $124.3 \pm 6.7$ & $111.8 \pm 6.2$ & $113.3 \pm 5.9$ & $93.6 \pm 3.0$ & $114.9 \pm 13.9$ & $126.0 \pm 8.2$ \\
VSL & $109.7 \pm 5.7$ & $89.1 \pm 5.6$ & $92.0 \pm 5.2$ & $73.5 \pm 2.1$ & $92.6 \pm 10.6$ & $104.1 \pm 7.2$ \\
VCL & $190.9 \pm 16.0$ & $195.2 \pm 10.7$ & $188.8 \pm 12.9$ & $163.2 \pm 7.7$ & $198.7 \pm 28.1$ & $206.1 \pm 14.0$ \\
ALH & $6.9 \pm 0.7$ & $8.0 \pm 0.3$ & $7.0 \pm 0.5$ & $6.7 \pm 0.4$ & $7.8 \pm 1.0$ & $7.8 \pm 0.4$ \\
BCF & $29.9 \pm 2.4$ & $21.3 \pm 3.0$ & $26.9 \pm 3.8$ & $24.5 \pm 3.2$ & $24.9 \pm 3.2$ & $25.0 \pm 2.9$ \\
STR & $87.2 \pm 0.5$ & $78.8 \pm 1.2$ & $79.8 \pm 1.6$ & $76.8 \pm 2.4$ & $80.0 \pm 2.6$ & $82.0 \pm 1.3$ \\
LIN & $59.3 \pm 2.2$ & $47.2 \pm 1.2$ & $50.5 \pm 1.9$ & $46.2 \pm 1.9$ & $50.0 \pm 4.1$ & $52.0 \pm 1.3$ \\
Area & $5.5 \pm 0.5$ & $5.5 \pm 0.5$ & $5.5 \pm 0.6$ & $6.0 \pm 0.6$ & $5.3 \pm 0.5$ & $5.4 \pm 0.5$ \\
\hline
\end{tabular}

*Sperm parameters measured by CASA were motility (\%), progressive motility (\%), velocity distribution (rapid, medium and slow \%), path velocity (VAP $\mu \mathrm{m} / \mathrm{s}$ ), progressive velocity (VSL $\mu \mathrm{m} / \mathrm{s}$ ), track speed (VCL $\mu \mathrm{m} / \mathrm{s}$ ), lateral amplitude (ALH $\mu \mathrm{m})$, beat cross frequency (BCF Hz), straightness (STR \%), linearity (LIN \%), and sperm head size (Area, $\mu \mathrm{m}$ ).

ranged from $\sim 40 \%$ to $75 \%$. In the current study, the percentage of X-bearing sperm ranged from $\sim 44 \%$ to $62 \%$, which is closer to range of $\sim 39 \%$ to $58 \%$ reported by Checa et al. [6]. Chandler et al. [11] evaluated the effects of semen collection frequency on semen sex ratio and reported the percentage of Y-bearing sperm changed in a sinusoid fashion over a period of 13.5 days. If the ratio of X- to Y-bearing sperm varies in a sinusoid fashion, that might explain the differences in semen sex ratios reported by various studies [4]-[6] [11]. If this were the case, then frequent semen collections would be necessary to establish the fluctuation pattern and determine when the peak variation in semen sex ratio occurs. Variation in semen sex ratio may also be attributed to stress; chronic stress has been shown to alter the gender ration toward females (for review, see Navara [12]). In mice, scrotal heat stress prior to mating results in fewer males born [13]. In the current study, 34 of 36 ejaculates of semen evaluated had a bias toward X-bearing sperm. The semen ejaculates were collected during late summer (August and September) when the bulls had already been exposed to heat stress for about 2 months.

The ratio of X- to Y-bearing sperm was found to differ among bulls, but not among ejaculates within bulls. These results are in agreement with Checa et al. [6] who reported a bull effect in the variation in X-bearing sperm in semen, but no ejaculate effect within bulls. In contrast, Chandler et al. [5] reported little variation among bulls in the ratio of $\mathrm{X}$ - to Y-bearing sperm, but differences among ejaculates within bulls. Sex ratio distortion has been reported within cattle families, and has been attributed to recombination rate of X-bearing sperm [14]. Therefore, it would be expected that not only ejaculates, but also individual bulls contribute to an altered sex ratio. Apparently, breed can also contribute to an altered sex ratio. Pauciullo et al. [15] compared the ratio of X- to Y-bearing sperm of bulls representing two minor breeds (Modicana and Agerolese) of cattle. The ratio of X-and $\mathrm{Y}$-bearing sperm was close to 1:1 in the Modicana breed, while the Y-fraction was about $4.5 \%$ higher than the $\mathrm{X}$-fraction in the Agerolese breed. Only bulls in the Agerolese breed showed significant altered sex ratio between bulls.

Another objective of this study was to determine if CASA sperm parameters could be used to predict an altered ratio of X- to Y-bearing sperm in semen collections. There has been speculation for years that Y-bearing sperm may have more a rapid motility than X-bearing sperm. If so, then a swim-up procedure should result in enrichment of Y-bearing sperm. However, swim-up of human sperm for intervals ranging from 15 to 150 min failed to alter the ratio of X- to Y-bearing sperm [16]. Furthermore, analysis of live births after assisted reproductive procedures indicate that sperm from male patients that sired male offspring actually had slower curvilinear and average path velocities than patients siring female offspring [17] [18].

No significant correlations were found between CASA parameters and percentage of X-chromosome bearing sperm across bulls. Different combinations of CASA and morphological parameters were found to correlate with the percentage of $\mathrm{X}$-bearing sperm within 3 of 6 bulls. Unfortunately, the prediction equations differed among the 
bulls and are not likely be of use in identifying ejaculates of semen with altered sex ratio. A possible sperm parameter that might merit further investigation for predicting the sex ratio of semen is acrosomal integrity. Chandler et al. [5] found acrosomal integrity (\% intact acrosomes) after $3 \mathrm{~h}$ incubation at $37^{\circ} \mathrm{C}$ post-thaw was positively correlated the percentage of Y-bearing sperm in semen and percentage of bull calves at birth. A study utilizing a double swim-up procedure with bull semen reported that the fraction of Y-bearing sperm is increased in some ejaculates following the double swim-up which was attributed to differences in capacitation of X-and Y-bearing sperm [19].

\section{Conclusion}

In summary, these results confirm that the ratio of X- to Y-bearing sperm may be skewed in some ejaculates of bull semen but this difference was attributed to differences among bulls rather than ejaculates within bulls. Some sperm parameters measured by CASA were associated with semen sex ratio but were not consistent across bulls and were unlikely to be of use in predicting the sex ratio of semen.

\section{Acknowledgements}

The authors acknowledge the University of Arkansas Agricultural Experiment Station and the Department of Animal Science for their financial support.

\section{References}

[1] Clutton-Brock, T H. and Iason, G R. (1986) Sex Ratio Variation in Mammals. The Quarterly Review of Biology, 61, 339-374. http://dx.doi.org/10.1086/415033

[2] Hardy, I.C.W. (1997) Possible Factors Influencing Vertebrate Sex Ratios: An Introductory Overview. Applied Animal Behaviour Science, 51, 217-241. http://dx.doi.org/10.1016/S0168-1591(96)01106-9

[3] Krackow, S. (1995) Potential Mechanisms for Sex Ratio Adjustment in Mammals and Birds. Biological Reviews, 70, 225-241. http://dx.doi.org/10.1111/j.1469-185X.1995.tb01066.x

[4] Chandler, J.E., Steinholt-Chenervet, H.C., Adkinson, R.W. and Moser, E.B. (1998) Sex Ratio Variation between Ejaculates within Sire Evaluated by Polymerase Chain Reaction, Calving, and Farrowing Records. Journal of Dairy Science, 81, 1855-1867. http://dx.doi.org/10.3168/jds.S0022-0302(98)75756-X

[5] Chandler, J.E., Taylor, T.M., Canal, A.L., Cooper, R.K., Moser, E.B., McCormick, M.E., Willard, S.T., Rycroft, H.E. and Gilbert, G.R. (2007) Calving Sex Ratio as Related to the Predicted Y-Chromosome-Bearing Spermatozoa Ratio in Bull Ejaculates. Theriogenology, 67, 563-571. http://dx.doi.org/10.1016/j.theriogenology.2006.09.006

[6] Checa, M.L., Dunner, S. and Canon, J. (2002) Prediction of X and Y Chromosome Content in Bovine Sperm by Using DNA Pools through Capillary Electrophoresis. Theriogenology, 58, 1579-1586. http://dx.doi.org/10.1016/S0093-691X(02)01077-4

[7] Coetzee, K., Kruger, T.F. and Lombard, C.J. (1999) Repeatability and Variance Analysis on Multiple Computer Assisted (IVOS) Sperm Morphology Readings. Andrologia, 31, 163-168. http://dx.doi.org/10.1046/j.1439-0272.1999.00257.x

[8] Gravance, C.G., Garner, D.L., Pitt, C., Vishwanath, R., Sax-Gravance, S.K. and Casey, P.J. (1999) Replicate and Technician Variation Associated with Computer Aided Bull Sperm Head Morphometry Analysis (ASMA). International Journal of Andrology, 22, 77-82. http://dx.doi.org/10.1046/j.1365-2605.1999.00148.x

[9] Campbell, R.C., Dott, H.M. and Glover, T.D. (1956) Nigrosin Eosin as Stain for Differentiating Live and Dead Sperm. The Journal of Agricultural Science, 48, 1-8. http://dx.doi.org/10.1017/S002185960003029X

[10] Heyen, D.W., Beever, J.E., Da, Y., Evert, R.E., Green, C., Bates, S.R.E., Ziegle, J.S. and Lewin, H.A. (1997) Exclusion Probabilities of 22 Bovine Microsatelite Markers in Fluorescent Multiplexes for Semi-Automated Parentage Testing. Animal Genetics, 28, 21-27. http://dx.doi.org/10.1111/j.1365-2052.1997.t01-1-00057.x

[11] Chandler, J.E., Canal, A.M., Paul, J.B. and Moser, E.B. (2002) Collection Frequency Affects Percent Y-Chromosome Bearing Spermatozoa, Sperm Head Area and Quality of Bovine Ejaculates. Theriogenology, 57, 1327-1346. http://dx.doi.org/10.1016/S0093-691X(01)00721-X

[12] Navara, K.J. (2010) Programming of Offspring Sex Ratios by Maternal Stress in Humans: Assessment of Physiological Mechanisms Using a Comparative Approach. Journal of Comparative Physiology B, 180, 785-796. http://dx.doi.org/10.1007/s00360-010-0483-9

[13] Pérez-Crespo, M., Pintado, B. and Gutiérrez-Adán, A. (2008) Scrotal Heat Stress Effects on Sperm Viability, Sperm DNA Integrity, and the Offspring Sex Ratio in Mice. Molecular Reproduction and Development, 75, 40-47. 
http://dx.doi.org/10.1002/mrd.20759

[14] Szyda, J., Simianer, H. and Lien, S. (2000) Sex Ratio Distortion in Bovine Sperm Correlates to Recombination in the Pseudoautosomal Region. Genetics Research, 75, 53-59.

[15] Pauciullo, A., Nicodemo, D., Peretti ,V., Marino, G., Iannuzzi, A., Cosenza, G., Di Meo, G.P., Ramunno, L., Iannuzzi, L., Rubes, J. and Di Berardino, D. (2012) X-Y Aneuploidy Rate in Sperm of Two “Minor” Breeds of Cattle (Bos taurus) by Using Dual Color Fluorescent in Situ Hybridization (FISH). Theriogenology, 78, 688-695. http://dx.doi.org/10.1016/j.theriogenology.2012.03.017

[16] Yan J., Feng, H.L., Chen, Z.J., Hu, J., Gao, X. and Qin, Y. (2006) Influence of Swim-Up Time on the Ratio of X- and Y-Bearing Spermatozoa. European Journal of Obstetrics \& Gynecology and Reproductive Biology, 129, 150-154. http://dx.doi.org/10.1016/j.ejogrb.2006.02.020

[17] Balli, K.S., Patton, W.C., Jacobsen, J.D., Corselli, J., King A. and Chan, P.J. (2004) Sperm Velocity in Seminal Plasma and the Association with Gender of Offspring. Systems Biology in Reproductive Medicine, 50, 37-40. http://dx.doi.org/10.1080/01485010490250560

[18] Maligaya, M.L., Chan, C.A., Jacobson, J.D., Patton, W.C., Corselli, J. and Chan, P.J. (2006) A Follow-Up Expanded Study of the Correlation of Sperm Velocity in Seminal Plasma and Offspring Gender. Archives of Andrology, 52, 3944.

[19] Madrid-Bury, N., Fernández, R., Jiménez, A., Pérez-Garnelo, S., Moreira, P.N., Pintado, B., Fuente, J. and GutiérrezAdán, A. (2003) Effect of Ejaculate, Bull, and a Double Swim-Up Sperm Processing Method on Sperm Sex Ratio. Zygote, 11, 229-235. http://dx.doi.org/10.1017/S0967199403002272 
Scientific Research Publishing (SCIRP) is one of the largest Open Access journal publishers. It is currently publishing more than 200 open access, online, peer-reviewed journals covering a wide range of academic disciplines. SCIRP serves the worldwide academic communities and contributes to the progress and application of science with its publication.

Other selected journals from SCIRP are listed as below. Submit your manuscript to us via either submit@scirp.org or Online Submission Portal.
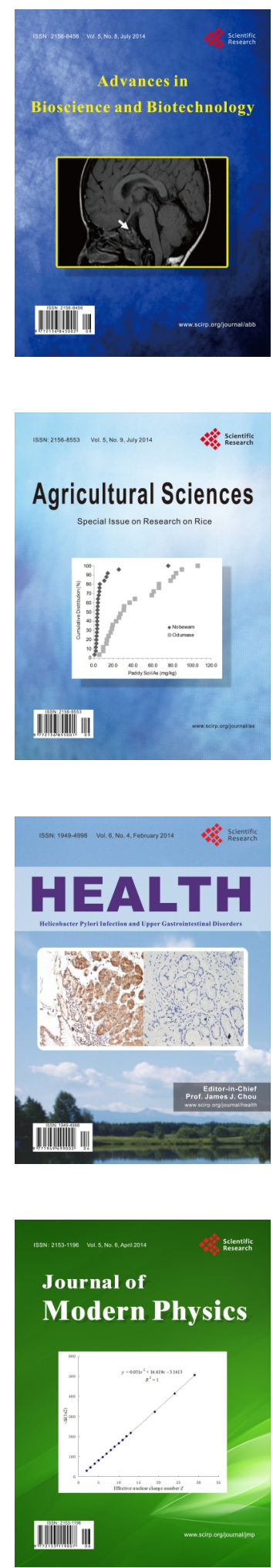
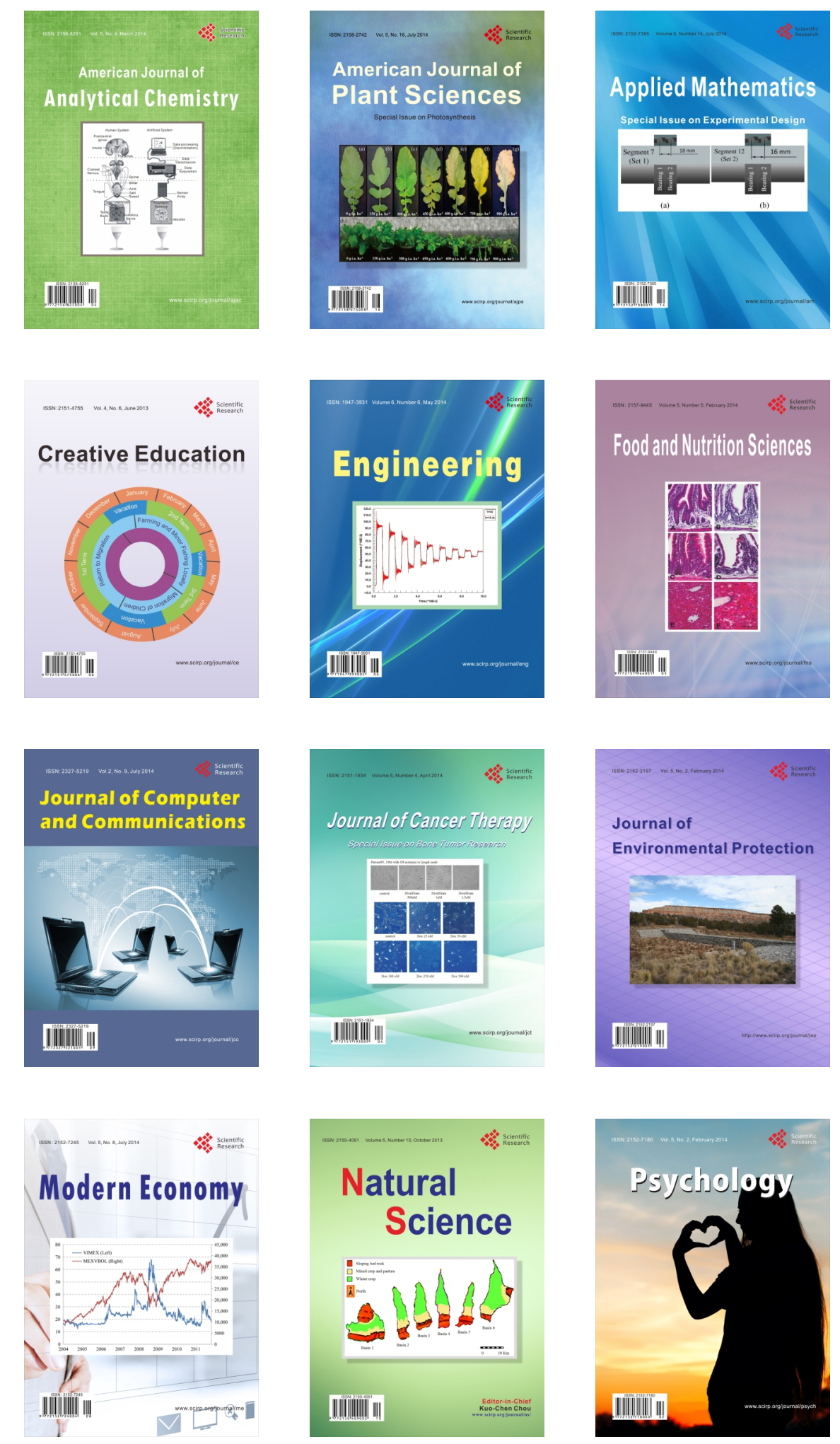